\title{
Tumor Necrosis Factor-Like Weak Inducer of Apoptosis Accelerates the Progression of Renal Fibrosis in Lupus Nephritis by Activating SMAD and p38 MAPK in TGF- $\beta 1$ Signaling Pathway
}

\author{
Zhiqin Liu, ${ }^{1}$ Leixi Xue, ${ }^{2}$ Zhichun Liu, ${ }^{2}$ Jun Huang, ${ }^{2}$ Jian Wen, ${ }^{2}$ Ji Hu, \\ Lin Bo, ${ }^{2}$ and Ru Yang ${ }^{2}$ \\ ${ }^{1}$ Department of Biological Science \& Engineering, Hebei University of Science \& Technology, Shijiazhuang 050018, China \\ ${ }^{2}$ Department of Rheumatology and Immunology, The Second Affiliated Hospital of Soochow University, Suzhou 215000, China
}

Correspondence should be addressed to Zhichun Liu; lzchun5190@sina.com

Received 26 November 2015; Revised 21 April 2016; Accepted 5 May 2016

Academic Editor: Hannes Neuwirt

Copyright (C) 2016 Zhiqin Liu et al. This is an open access article distributed under the Creative Commons Attribution License, which permits unrestricted use, distribution, and reproduction in any medium, provided the original work is properly cited.

\begin{abstract}
This study aim was to explore the effects of tumor necrosis factor-like weak inducer of apoptosis (TWEAK) in lupus nephritis and its potential underlying mechanisms. MRL/lpr mice were used for in vivo experiments and human proximal tubular cells (HK2 cells) were used for in vitro experiments. Results showed that MRL/lpr mice treated with vehicle solution or LV-Control shRNA displayed significant proteinuria and severe renal histopathological changes. LV-TWEAK-shRNA treatment reversed these changes and decreased renal expressions of TWEAK, TGF- $\beta 1$, p-p38 MAPK, p-Smad2, COL-1, and $\alpha$-SMA proteins. In vitro, hTWEAK treatment upregulated the expressions of TGF- $\beta 1$, p-p38 MAPK, p-SMAD2, $\alpha$-SMA, and COL-1 proteins in HK2 cells and downregulated the expressions of E-cadherin protein, which were reversed by cotreatment with anti-TWEAK mAb or SB431542 treatment. These findings suggest that TWEAK may contribute to chronic renal changes and renal fibrosis by activating TGF- $\beta 1$ signaling pathway, and phosphorylation of Smad2 and p38 MAPK proteins was also involved in this signaling pathway.
\end{abstract}

\section{Introduction}

The kidney is one of the most commonly affected organs in systemic lupus erythematosus (SLE); about half of the patients with SLE experience lupus nephritis (LN). Although the survival rate for patients with LN has improved to $88 \%$ over the last decade, approximately $10 \%-20 \%$ of these patients will develop end-stage renal disease (ESRD) [1]. Fibrotic lesions such as sclerosed glomeruli, interstitial fibrosis, and fibrous vessels are strongly associated with poor renal outcomes in LN. Therefore, the treatment of LN should aim to reduce proteinuria and improve the pathologic features responsible for glomerulosclerosis and tubulointerstitial fibrosis which eventually lead to ESRD, but here we want to stress that repeat biopsy in the clinical treatment is not necessary in most cases. Tubulointerstitial fibrosis is the final common manifestation of various chronic kidney diseases, and progressive accumulation of extracellular matrix (ECM) proteins including collagen $1(\mathrm{COL}-1)$ in the interstitial area is a key feature [2].

The interaction between the tumor necrosis factor (TNF) superfamily ligands and their receptors is central to the pathogenesis of SLE [3]. Tumor necrosis factor-like weak inducer of apoptosis (TWEAK) is one member of these superfamily ligands, which binds to its receptor, fibroblast growth factor-inducible-14 (Fn14) [4-8]. TWEAK has been shown to play important roles in cell proliferation and differentiation, cell apoptosis, inflammation, and fibrosis through a variety of intracellular signaling pathways, including the TGF- $\beta$ signaling pathway [8-10]. In kidney, TWEAK expression has been reported in mesangial cells, podocytes, endothelial cells, and tubular cells [11]. Increased leukocytes and urinary TWEAK levels are associated with renal disease activity in human $\mathrm{LN}[12,13]$. In addition to its 
well-established proinflammatory effects, TWEAK regulates matrix metalloproteinases (MMPs) and tissue inhibitors of MMPS (TIMPs) in myopathies and is associated with the intestinal damage linked to extracellular matrix- (ECM-) related proteins [14-16]. TWEAK-knockout mice were protected from kidney fibrosis in a unilateral ureteral obstruction (UUO) model [10], whereas overexpression of TWEAK gave rise to renal fibrosis in previously normal kidneys. Moreover, anti-Fn14 antibodies were found to reduce residual fibrosis in the acute phase of ischemia reperfusion in mice [17]. These results suggest that TWEAK may contribute to chronic kidney injury and renal fibrosis in LN. In addition, TWEAK-Fn14 interactions also play an important role in the pathogenesis of neuropsychiatric lupus [18, 19]. MRL/lpr Fn14 knockout mice showed significantly reduced neuron degeneration, hippocampal gliosis, and preserved blood brain barrier permeability $[18,19]$. So disrupting TWEAK-Fn14 interactions may be an effective approach to the treatment of SLE.

Transforming growth factor-beta (TGF- $\beta$ ) signaling, enhanced by proinflammatory cytokines, is a central inducer of renal fibrosis [20]. It has been reported that TGF- $\beta$ can augment TWEAK and Fn14 expression in inflammatory diseases [21]. Conversely, TWEAK enhanced TGF- $\beta 1$ expression in human bronchial epithelial cells and human retinal pigment epithelial cells [22]. Once TGF- $\beta$ is released, the biological activities of TGF- $\beta$ are mediated by activation of the canonical TGF- $\beta$ signaling pathway through binding its receptor 2 (TGF- $\beta$ R2), which then dimerizes with TGF- $\beta$ receptor 1 (TGF- $\beta$ R1) leading to intracellular phosphorylation and activation of the transcription factors Smad2 and Smad3 [23]. Activation of noncanonical TGF- $\beta$ signaling pathway also has been shown to contribute to TGF- $\beta$-induced renal fibrosis, specifically through the activation of p38 mitogenactivated protein kinase (MAPK) protein [24]. A previous report showed that the single blockade of p38 MAPK after the emergence of established fibrosis is effective in reducing subsequent renal fibrosis in the model of UUO [25]. Among the three isoforms of TGF- $\beta$, TGF- $\beta 1$ has been identified as the most potent mediator and convergent pathway in renal fibrosis [26].

However, the significance of TWEAK-TGF- $\beta$ signaling pathway in the progression of renal fibrosis of lupus nephritis remains to be determined. We hypothesize that TGF- $\beta 1$ is an important component of TWEAK-induced renal fibrosis in lupus nephritis. To verify our hypothesis, we have studied the role of TWEAK in the activation of TGF- $\beta 1$ through the canonical signaling pathways of TGF- $\beta$ and p38 MAPK in vivo and in vitro.

\section{Materials and Methods}

2.1. shRNA Design and Generation of Lentivirus-Based shRNA. Three pair of shRNAs targeting mice TWEAK gene (Genbank accession number: NM_011614) were designed using the siRNA Target Finder and Design Tool available at http://www.ambion.com/ and were commercially obtained from GeneChem (Shanghai, China). The sequences of these shRNAs were (a) TWEAK-shRNA1
5'-CGGTAACCTACTTTGGACT CTTTCCTCGAGGAAAGAGTCCAAAGTAGGTTATT TTT G- $3^{\prime}$ and reverse $5^{\prime}$-AATTCA AAAAAATACTTTGGACTCTTTCCTCGAGGAAAG AGTCCAAAGTAGGTTA- ${ }^{\prime}$, (b) TWEAKshRNA2 $5^{\prime}$-CCGGAATTTACAGTCAT CAGGGCTCTCGAGAGCCCTGATGACTGTAAATTCTTTTTG- ${ }^{\prime}$ and reverse $5^{\prime}$-AATTCAAAAAGAATTTACAGTCATCAGGGCTCTCGAGAGCCCTGATGACTGTAA ATTC-3' ${ }^{\prime}$, and (c) TWEAK shRNA3 $5^{\prime}$-CCGGCGAGCTATTGCAGCCCATT ATCTCGA GATAATGGGCTGCAATAGCTCGTTTTTG- ${ }^{\prime}$ and reverse $5^{\prime}$-AATTCAAAAACGAG CTATTGCAGCCCATTATCTCGAGATAATGGGCTGCAATAGCTCG-3'. Each of these fragments was amplified and ligated into the hU6-MCS-CMV-EGFP (GV115) vector. The nonsilencing shRNA control sequences (LV-Control shRNA) were also designed as a negative control for TWEAK-shRNA (LV-Control shRNA: $5^{\prime}$-CCGGTTC TCCGAACGTGTCACGTTTCAAGAGAACGTGACACGTTCGGAGAATT TTTG- $3^{\prime}$ and reverse $5^{\prime}$-AATTCAAAAATTCTCCGAACGTGTCACGTTCTCTTGAAACGTGACACG TTCGGAGAA- ${ }^{\prime}$ ). 293 T cells were used to detect the silencing efficiency of TWEAK gene by LVTWEAK-shRNA. TWEAK mRNA and protein levels were detected by Western blotting at 48-hour posttransfection. TWEAK-shRNA1 was the most effective suppressed TWEAK protein expression (results omitted). So we chose TWEAKshRNAl as the LV-TWEAK-shRNA for in vivo experiments. In this paper, the TWEAK-shRNA is referred to as the TWEAK-shRNA1.

2.2. Mice. Thirty-two 13-week-old female MRL/lpr mice were purchased from the Shanghai Slac Laboratory Animal Co., Ltd., and maintained at the Soochow University. Ten MRL/MPJ mice constituted the normal control group ( $n=$ 10). Animal study protocols were approved by the Animal Care Committee of Soochow University and the experiments were carried out according to the guidelines of the committee. Mice were injected in the tail vein with either $2 \times 10^{7} \mathrm{TU} \mathrm{LV}$ TWEAK-shRNA (experimental group, $n=11$ ), $2 \times 10^{7} \mathrm{TU}$ negative LV-Control shRNA (LV-Control shRNA group, $n=$ 11), or phosphate-buffered saline (vehicle control group, $n=$ 10) once. All animals were housed in the well-ventilated experimental animal center of Soochow University under specific pathogen-free conditions. Mice were provided ad libitum with food and water and maintained on a natural circadian cycle at $40-70 \%$ humidity and a temperature of $20-25^{\circ} \mathrm{C}$. All animals were sacrificed by cervical vertebral dislocation four weeks after injection.

2.3. Cell Culture. Human proximal tubular cells (HK2 cells) from the Cell Center of Soochow University, Suzhou, China, were grown in a $5 \% \mathrm{CO}_{2}$ atmosphere at $37^{\circ} \mathrm{C}$ in Dulbecco's modified Eagle's medium (DMEM) containing 10\% fetal bovine serum (FBS) in six-well plastic plates. Once cells reached approximately $80 \%$ confluence, they were serumstarved in DMEM containing 0.2\% FBS overnight before treatment with or without recombinant human TWEAK (hTWEAK, R\&D Systems, Minneapolis, MN, USA) at $100 \mathrm{ng} / \mathrm{mL}$ or with TGF- $\beta 1$ at $1 \mathrm{ng} / \mathrm{mL}$ (Sigma, St. Louis, MO, 
USA). In our previous studies, we have found that coculture with a concentration of $10 \mu \mathrm{g} / \mathrm{mL}$ anti-TWEAK mAb could functionally inhibit the role of TWEAK in cultured PBMC, so, in this study, we also cocultured the hTWEAK-stimulated HK2 cells with $10 \mu \mathrm{g} / \mathrm{mL}$ anti-TWEAK monoclonal antibody (mAb; R\&D Systems, Inc., USA), or with $10 \mu \mathrm{M}$ SB431542 (TGF- $\beta 1$ receptor kinase inhibitor, Sigma-Aldrich, Saint Louis, USA) or dimethyl sulfoxide alone. After $24 \mathrm{~h}$, the cells were collected and the expression of TWEAK, p-p38 MAPK, p-Smad2, TGF- $\beta 1$, alpha-smooth muscle actin ( $\alpha$ SMA), COL-1, and E-cadherin proteins in HK2 cells were detected by Western blotting.

2.4. Histopathology. The kidneys were dissected and fixed in $10 \%$ buffered formalin, and paraffin-embedded sections of kidney tissues ( $3 \mathrm{~mm}$ thick) were stained with hematoxylin and eosin (H\&E), periodic acid-Schiff (PAS), and Masson's trichrome for histopathological examinations. PAS-positive deposits were each scored from 0 to 4 ( 0 , absent; 1 , mild; 2 , mild-moderate; 3 , moderate; and 4, severe). The presence of inflammatory cell infiltrates determined by $\mathrm{H} \& \mathrm{E}$ staining and the quantification of Aniline Blue-positive areas (indicating fibrotic areas) determined by Masson's trichrome staining were each graded on a scale of 0 to 4 ( 0 , absent; 1 , present in $<25 \%$ of the section; 2 , present in $25-50 \%$ of the section; 3 , present in $50-75 \%$ of the section; and 4 , present in $>75 \%$ of the section).

2.5. Urine Analysis. To determine renal injury in $\mathrm{MRL} / \mathrm{lpr}$ mice, urine samples were collected at 17 weeks of age using metabolic cages for $24 \mathrm{~h}$. Urine protein concentrations were measured by a bicinchoninic acid (BCA) protein assay (Thermo Fisher, Waltham, MA, USA).

2.6. Western Blotting. The kidneys or cells were lysed with Nonidet P-40 (NP-40) lysis buffer as previously described [27]. Protein concentrations were determined with the BCA Protein Assay Kit (Thermo Fisher Scientific, Waltham, MA, USA). From each sample preparation, $60 \mu \mathrm{g}$ of total proteins was mixed in Laemmli loading buffer, boiled for $5 \mathrm{~min}$, separated by $10 \%$ SDS-PAGE, and then transferred to PVDF blotting membranes (Millipore, MA, USA). Membranes were blocked with Tris-buffered saline-Tween/1\% nonfat dry milk and incubated overnight at $4^{\circ} \mathrm{C}$ with goat antibodies specific for TWEAK (1:500, Santa Cruz, CA, USA), rabbit antibodies specific for p-p38 MAPK and p38 MAPK (1:500; Santa Cruz, CA, USA), goat antibodies specific for TGF- $\beta 1$ (1:250; Santa Cruz, CA, USA), mouse antibodies specific for COL-1, $\alpha$-SMA, and E-cadherin (1:500; Abcam, Cambridge, $\mathrm{UK}$ ), rabbit antibodies specific for $\mathrm{p}-\mathrm{Smad} 2$ and Smad2 (1:500; Abcam, Cambridge, UK), and rabbit polyclonal antibody to $\beta$-actin $(1: 1000$; Sigma Aldrich, Saint Louis, USA). After a final incubation with a $1: 10,000$ dilution of horseradish peroxidase-conjugated secondary antibodies (Zhongshan Biotechnology, Beijing, China) for $2 \mathrm{~h}$ at room temperature, the membranes were developed with an ECL detection system (Santa Cruz Biotechnology, CA, USA) and exposed to X-ray film from $30 \mathrm{~s}$ to $3 \mathrm{~min}$ to visualize chemiluminescent proteins.
Each sample was run in triplicate. The levels of TWEAK, TGF- $\beta 1$, p-p38 MAPK, p-smad2, COL- $1, \alpha$-SMA, and Ecadherin were evaluated by a FluorChem FC2 system (NatureGene Corp., New Jersey, USA). Data are expressed as the ratio of TWEAK, TGF- $\beta 1, \mathrm{COL}-1, \alpha$-SMA, and Ecadherin band integral optical density (IOD) and $\beta$-actin IOD, the ratio of p-p38 MAPK IOD and p38 MAPK IOD, or the ratio of $\mathrm{p}$-smad2 IOD and smad2 IOD in the same samples.

2.7. Immunohistochemistry Staining of Kidney Sections. Immunohistochemistry was performed on kidney paraffin sections with goat antibodies specific for Fn14 (1:300, Santa Cruz, CA, USA), goat antibodies specific for TWEAK (1:500, Santa Cruz, CA, USA), mouse antibodies specific for COL-1 and $\alpha$-SMA (1:500; Abcam, Cambridge, UK) using the Streptavidin-Biotin Complex (SABC) immunohistochemical technique (SABC kit; Boster Biological Technology Ltd., Wuhan, China). Mean histologic score for each mouse was obtained in a blinded manner in glomerular (20 random glomeruli) and interstitial (20 random fields) sections and each was graded on a scale of 0 to 4 ( 0 , absent; 1 , present in $<25 \%$ of the section; 2 , present in $25-50 \%$ of the section; 3 , present in $50-75 \%$ of the section; and 4 , present in $>75 \%$ of the section) [28].

2.8. Statistical Analysis. All data were expressed as mean \pm standard deviation. A one-way analysis of variance (ANOVA) test was used for comparison of more than two groups. The differences between the groups were assessed with the post hoc Bonferroni test. The datasets were analyzed using the SPSS v 13.0 statistical package. Each experiment was repeated at least 3 times to assess reproducibility. A $p$ value of 0.05 was considered as statistically significant.

\section{Results}

3.1. LV-TWEAK-shRNA Treatment Alleviated Histopathological Changes in Kidneys of MRL/lpr Mice. Only one mouse in the vehicle control group died during the study. Compared to age-matched MRL/MPJ mice, MRL/lpr mice showed large amounts of lymphocyte invasion, mesangial and glomerular endothelial cell proliferation, glomerular sclerosis, and casts in the tubules, which was accompanied by accumulation of numerous fibroblasts in the renal interstitial area and glomerular area and around the blood vessels (Figure 1). Histological assessment blindly scored by a pathologist revealed moderate to severe glomerular damage and signs of perivascular inflammation in all of the vehicle control and LV-Control shRNA treated mice. These pathological changes were associated with a significant increase in TWEAK protein expression in the kidneys (Figure 2) and elevated proteinuria levels (Table 1). In contrast, the LV-TWEAK-shRNA treated MRL/lpr mice showed a much milder degree of focal glomerular cell proliferation, inflammatory cell infiltration, and fibroblast accumulation in the kidneys. Besides, capillary wall thickening with PAS-positive material deposition was apparently attenuated (Figure 1). TWEAK protein expression 
TABLE 1: Proteinuria in MRL/MPJ and MRL/lpr mice (mg/24 h).

\begin{tabular}{|c|c|c|c|c|}
\hline & Control & Control shRNA & TWEAK-shRNA & MRL/MPJ \\
\hline Urine protein (13 weeks) & $5.12 \pm 0.73$ & $5.34 \pm 0.69$ & $5.51 \pm 1.03$ & $0.97 \pm 0.19$ \\
\hline Urine protein (17 weeks) & $7.68 \pm 1.51^{\# *}$ & $7.61 \pm 1.48^{\#}$ & $3.16 \pm 0.37^{\# \Delta}$ & $1.25 \pm 0.19$ \\
\hline
\end{tabular}

Control: MRL/lpr mice treated with PBS; Control shRNA: MRL/lpr mice treated with LV-Control shRNA; TWEAK-shRNA: MRL/lpr mice treated with LVTWEAK-shRNA; MRL/MPJ: MRL/MPJ mice as normal control. Data are mean \pm standard deviation. ${ }^{\#} p<0.05$ versus MRL/MPJ at the same time point, ${ }^{\triangle} p<0.05$ versus control MRL/lpr mice at the same time point, and ${ }^{*} p<0.05$ versus control MRL/lpr mice at 13 weeks of age.

in the kidney and proteinuria also decreased significantly (Figure 2 and Table 1).

\subsection{Distribution of TWEAK and Fn14 Proteins in Kidneys} of MRL/lpr Mice. In this study, the immunohistochemistry results showed that TWEAK and Fn14 proteins were mainly distributed in the glomerular area, tubules, and inflammatory cells of the kidneys in MRL/lpr mice but were only weakly observed in kidneys of MRL/MPJ mice (Figure 3).

3.3. Effects of LV-TWEAK-shRNA Treatment on the Expressions of $\alpha$-SMA and COL-1 Proteins in Kidneys of MRL/lpr Mice. It is well known that fibroblast proliferation, altered expression, and overdeposition of extracellular matrix contribute to progressive renal fibrosis. Collagen overproduction, including COL-1, is the major contributor to renal fibrosis [29]. Myofibroblasts in the kidney have also been considered to represent an activated population of resident fibroblasts, which are commonly identified by their expression of $\alpha$-SMA [30]. So we also investigated the role of TWEAK on $\alpha$-SMA and COL-1 expressions in kidneys of MRL/lpr mice.

Compared with MRL/MPJ mice, control MRL/lpr mice treated with vehicle showed higher expression levels of COL- 1 and $\alpha$-SMA proteins in kidneys by Western blotting and immunohistochemistry; however, LV-TWEAK-shRNA treatment of MRL/lpr mice significantly decreased expression levels of COL- 1 and $\alpha$-SMA proteins in kidneys. LV-Control shRNA treatment had no effect on $\alpha$-SMA and COL-1 protein expressions (Figures 4(a) and 4(b)).

3.4. Effects of LV-TWEAK-shRNA Treatment on Expressions of TGF- $\beta 1$, p-p38 MAPK, and p-Smad2 Proteins in Kidneys of MRL/lpr Mice. To investigate the effects of TWEAK on TGF- $\beta$ signaling pathway, we determine the expression levels of TGF- $\beta 1$ and its associated downstream signaling pathway proteins (p-p38 MAPK and p-Smad2) in kidneys of MRL/lpr mice after LV-TWEAK-shRNA treatment.

Compared with MRL/MPJ mice, MRL/lpr mice had higher expression level of TGF- $\beta 1$ protein and stronger phosphorylation degree of Smad 2 and p38 MAPK proteins in kidneys of control MRL/lpr mice. After LV-TWEAK-shRNA treatment, expression level of TGF- $\beta 1$ and phosphorylation degree of Smad2 and p38 MAPK proteins all decreased in MRL/lpr mice, but LV-Control shRNA treatment had no similar effects (Figure 5).

3.5. Effects of SB431542 on hTWEAK Induced Expressions of $\alpha$-SMA, COL-1, and E-Cadherin Proteins in HK2 Cells. E-Cadherin which is localized on the surface of epithelial cells plays an important role in epithelial integrity, and the expression of E-cadherin decreases during the process of renal interstitial fibrosis [31]. So we also show expression of E-cadherin in HK2 cells.

After treatment of HK2 cells with hTWEAK for $24 \mathrm{~h}$, the expression levels of $\alpha$-SMA and COL-1 proteins increased significantly, whereas E-cadherin protein expression levels decreased significantly; anti-TWEAK mAb cotreatment diminished the increase of $\alpha$-SMA and COL-1 protein expressions and upregulated E-cadherin protein expression. SB431542, a TGF- $\beta$ receptor 1 inhibitor, showed the same effect as anti-TWEAK $m A b$ and reversed the upregulation of $\alpha$-SMA and COL-1 protein expressions and the downregulation of E-cadherin protein expressions induced by hTWEAK in HK2 cells (Figure 6).

3.6. Effects of SB431542 on hTWEAK Induced Expressions of TGF- $\beta 1$, p-Smad2, and $p-p 38$ MAPK Proteins in HK2 Cells. As shown in Figure 7, TGF- $\beta 1$ culture of HK2 cells induced significant phosphorylation of Smad2 and p38 MAPK proteins. hTWEAK treatment had the same effect on phosphorylation of Smad2 and p38 MAPK proteins in HK2 cells and upregulated TGF- $\beta 1$ protein expression. However, anti-TWEAK $m A b$ treatment inhibited upregulation of TGF$\beta 1$ protein and phosphorylation of Smad2 and p38 MAPK proteins induced by hTWEAK treatment. TGF- $\beta$ receptor 1 inhibitor SB431542 also blocked phosphorylation of Smad2 and $\mathrm{p} 38$ MAPK proteins induced by hTWEAK treatment (Figure 7).

\section{Discussion}

MRL/lpr mice are one of the commonly used animal models that show pathologies resembling human SLE, including significant amounts of serum autoantibodies along with immune complex glomerulonephritis and vasculitis [32]. Our experiments demonstrated that MRL/lpr mice at the age of 13 weeks presented with large amounts of proteinuria. At 17 weeks age, the MRL/lpr mice presented with more proteinuria and severe renal pathological changes, such as infiltration of inflammatory cells, collagen deposition, and immune complexes deposition in the capillary wall and basement membrane, which were consistent with the results of other researchers. Moreover, glomerulosclerotic lesions were also demonstrated [33].

TWEAK mRNA level was initially reported to be increased in mouse and human with acute (induced by lipopolysaccharide) or chronic (autoimmune pathologies) inflammatory processes like lupus erythematosus [34]. In 

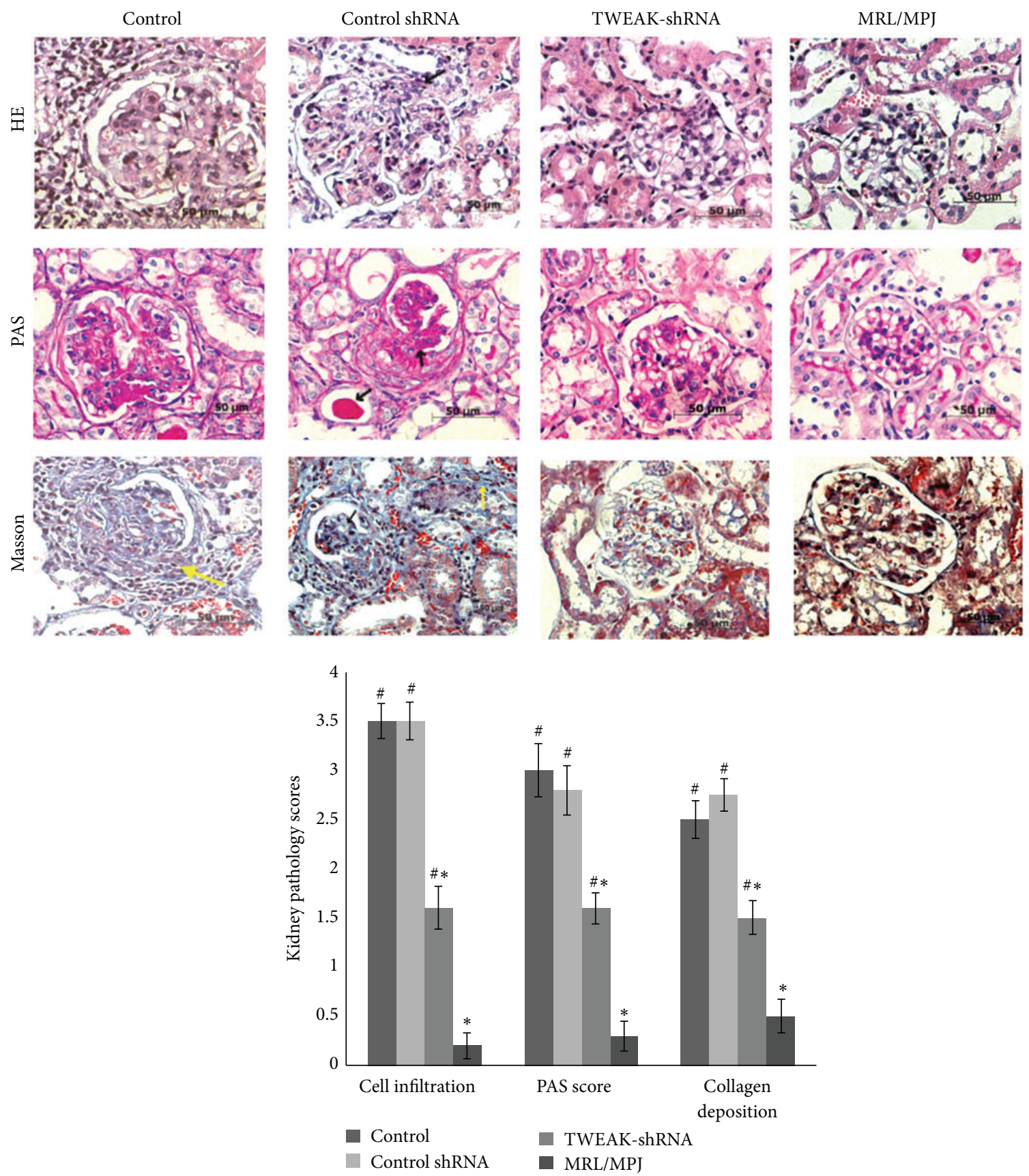

FIGURE 1: LV-TWEAK-shRNA treatment alleviated histopathological changes in kidneys of MRL/lpr mice. HE representative hematoxylin and eosin- (H\&E-) stained sections of formalin-fixed kidneys. PAS representative micrographs of periodic acid-Schiff- (PAS-) stained sections from paraffin-embedded tissues. Masson representative micrographs of Masson-stained sections of paraffin-embedded tissue of the glomerular and interstitial. The kidney pathological scores of cell infiltration and PAS-positive and collagen deposition were shown at the bottom. Compared with those from MRL/MPJ mice, the kidneys from MRL/lpr mice which were treated with PBS or LV-Control shRNA had severe inflammatory infiltrates and histopathology changes, such as a large PAS-positive deposit in the glomeruli (black arrows), segmental overlying cellular crescent sclerosis, crescents, and periglomerular infiltrates, and accumulation of numerous fibroblasts (yellow arrows); damaged kidney tubules and blood vessels were also evident. The kidneys from MRL/MPJ mice showed intact glomerular and tubulointerstitial structures and very few fibroblasts. Control: MRL/lpr mice treated with PBS; Control shRNA: MRL/lpr mice treated with LV-Control shRNA; TWEAK-shRNA: MRL/lpr mice treated with LV-TWEAK-shRNA; and MRL/MPJ: MRL/MPJ mice as normal control. The error bars represent the standard deviation. ${ }^{\#} p<0.05$ versus MRL/MPJ mice and ${ }^{*} p<0.05$ versus control MRL/lpr mice. Scale bar: $50 \mu \mathrm{m}$. 


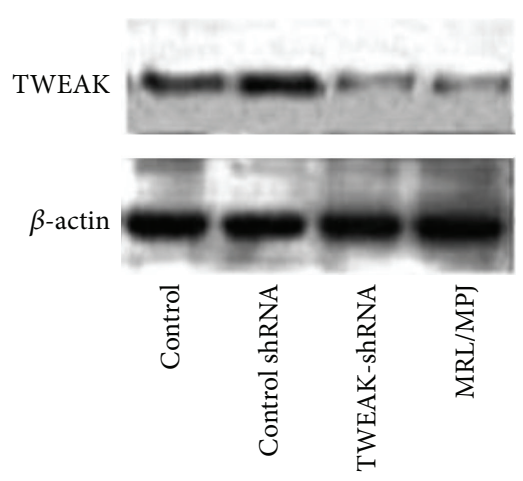

(a)

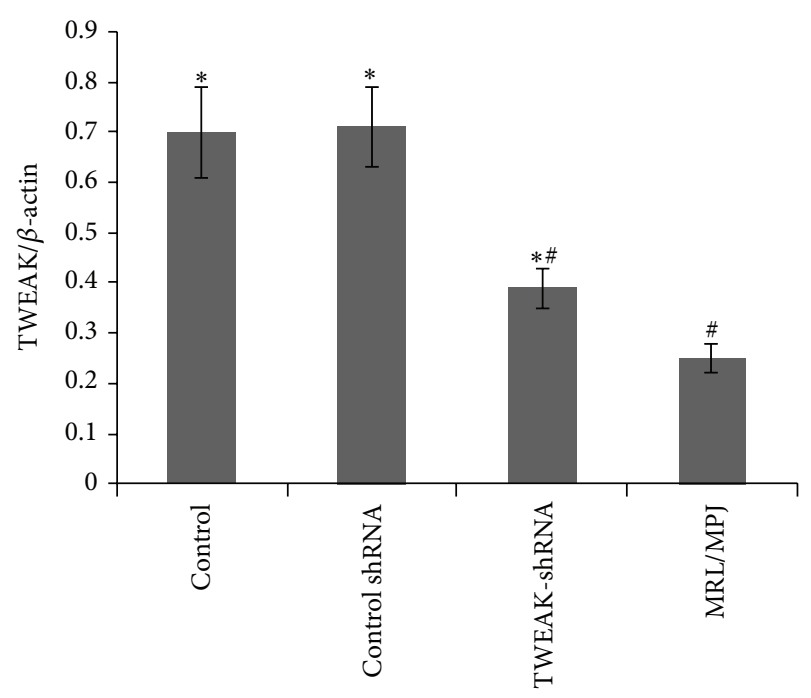

(b)

FIGURE 2: The expression of TWEAK protein in the kidneys. Total lysates of kidneys were collected for Western blotting to measure TWEAK protein expression. The representative experiment was presented (a). The ratios of TWEAK/ $\beta$-actin IOD were shown (b). Control: MRL/lpr mice treated with PBS; Control shRNA: MRL/lpr mice treated with LV-Control shRNA; TWEAK-shRNA: MRL/lpr mice treated with LVTWEAK-shRNA; and MRL/MPJ: MRL/MPJ mice as normal control. The error bars represent the standard deviation. ${ }^{*} p<0.05$ versus MRL/MPJ mice and ${ }^{\#} p<0.05$ versus control MRL/lpr mice.
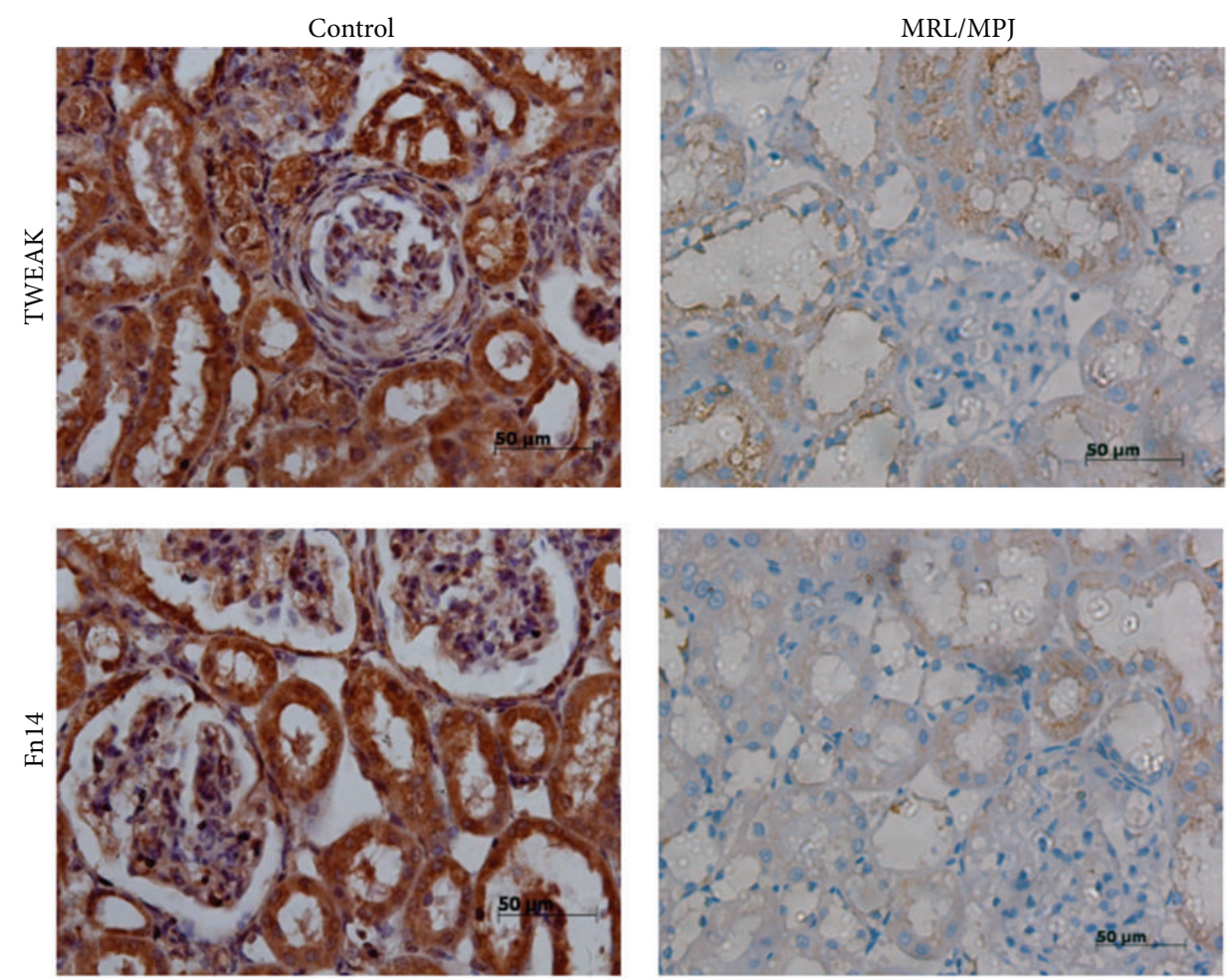

FIGURE 3: Distribution of TWEAK and Fn14 in kidneys of MRL/Lpr mice. Immunohistochemistry staining of kidneys shows strong expression of TWEAK and Fn14 in the kidneys from MRL/lpr mice, but they were only weakly seen in the kidney of MRL/MPJ mice. Control: MRL/lpr mice treated with PBS and MRL/MPJ: MRL/MPJ mice as normal control. Scale bar: $50 \mu \mathrm{m}$.

contrast to the other TNF ligand-receptor pairs, TWEAK and Fn14 are evidently a monogamous ligand-receptor pair [5]. In this study, we found that TWEAK and Fn14 were weakly expressed in normal condition, while they significantly increased in MRL/lpr mice; the serious pathological changes in the kidneys from MRL/lpr mice were associated with significantly increased renal TWEAK protein. LV-TWEAKshRNA treatment significantly decreased TWEAK protein 

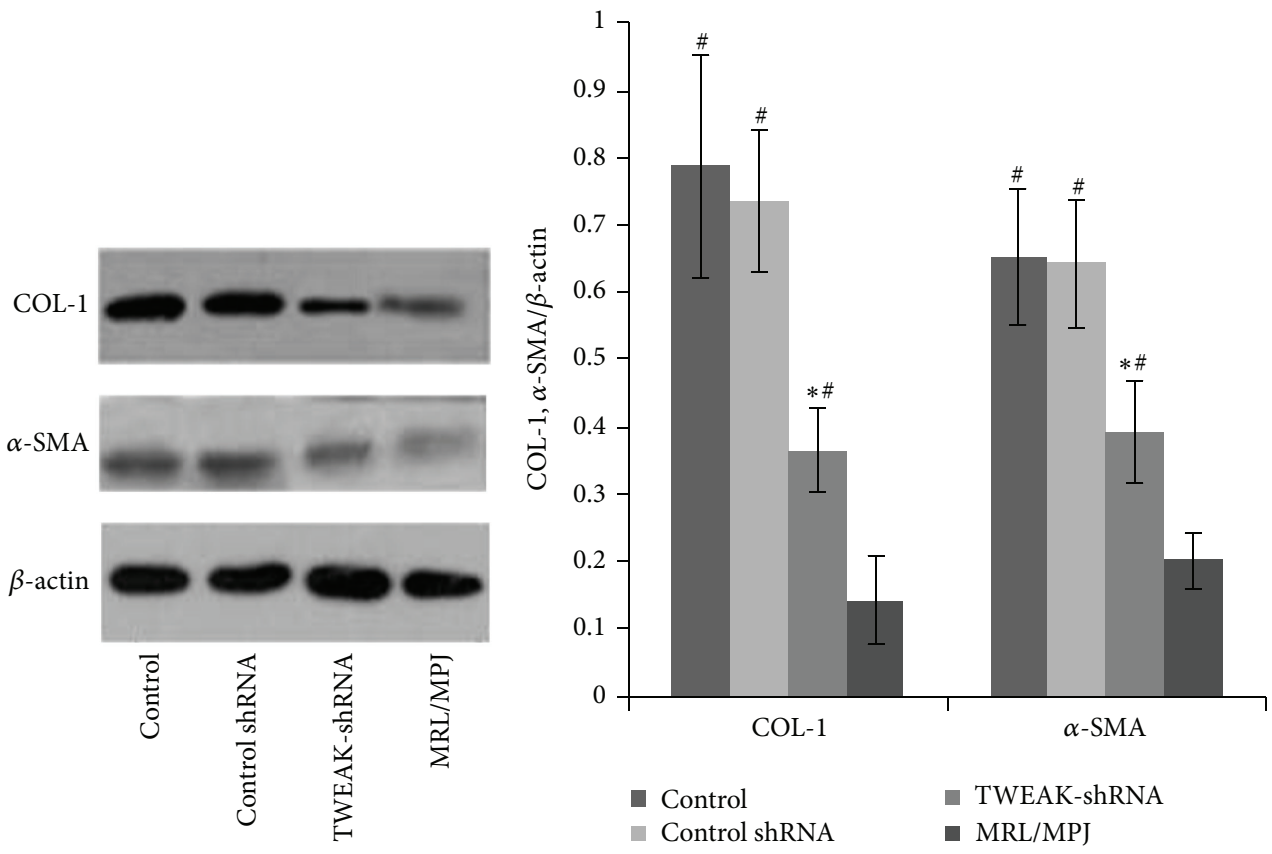

(a)
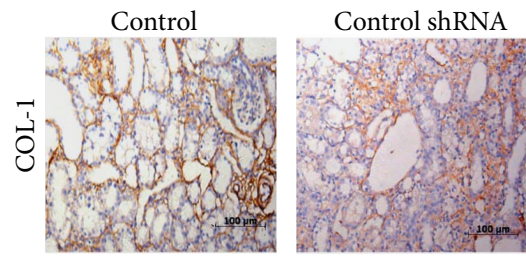

TWEAK-shRNA

$\mathrm{MRL} / \mathrm{MPJ}$
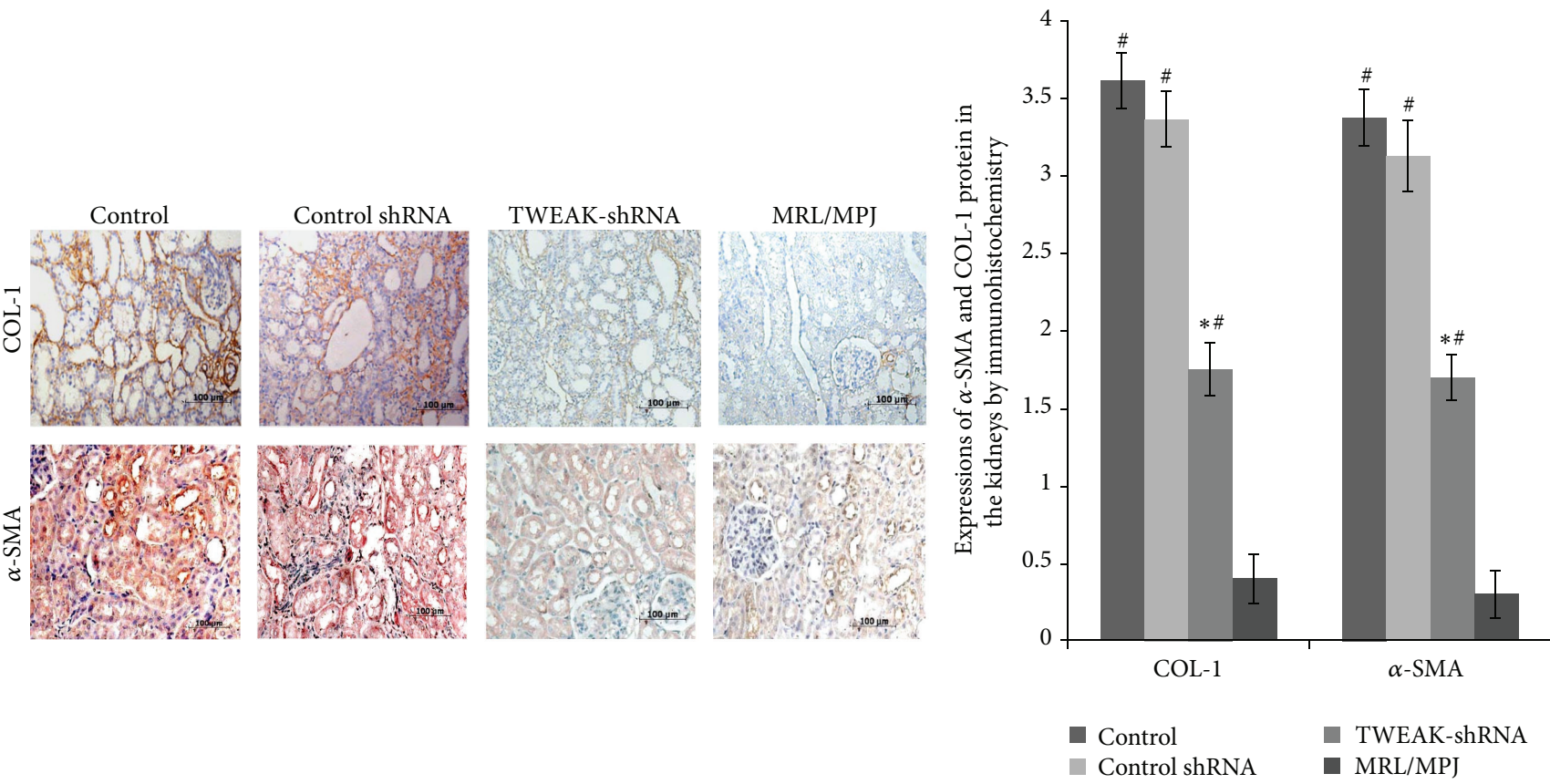

(b)

Figure 4: Effects of LV-TWEAK-shRNA treatment on expressions of $\alpha$-SMA and COL-1 proteins in kidneys of MRL/lpr mice. (a) Total lysates of kidneys were collected for Western blotting to measure COL- 1 and $\alpha$-SMA protein expressions. The representative experiment was presented (left). The ratios of COL- $1 / \beta$-actin IOD and $\alpha$-SMA/ $\beta$-actin IOD were shown (right). (b) Distribution of $\alpha$-SMA and COL- 1 proteins in the kidney was tested by immunohistochemistry. The histologic scores of $\alpha$-SMA and COL-1 proteins were shown at the bottom. Control: MRL/lpr mice treated with PBS; Control shRNA: MRL/lpr mice treated with LV-Control shRNA; TWEAK-shRNA: MRL/lpr mice treated with LV-TWEAK-shRNA; and MRL/MPJ: MRL/MPJ mice as normal control. The error bars represent the standard deviation. ${ }^{*} p<0.01$ versus MRL/MPJ mice and ${ }^{*} p<0.01$ versus control MRL/lpr mice. Scale bar: $100 \mu \mathrm{m}$. 


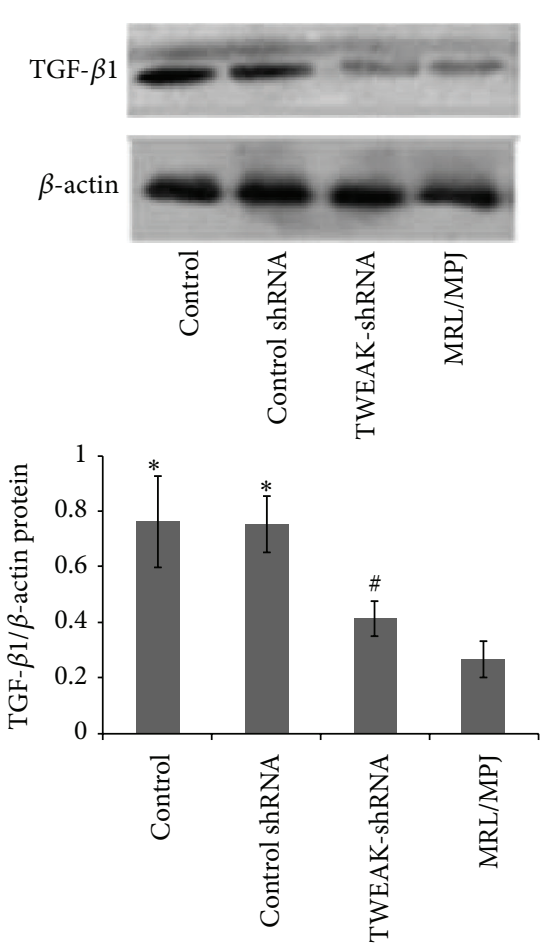

(a)

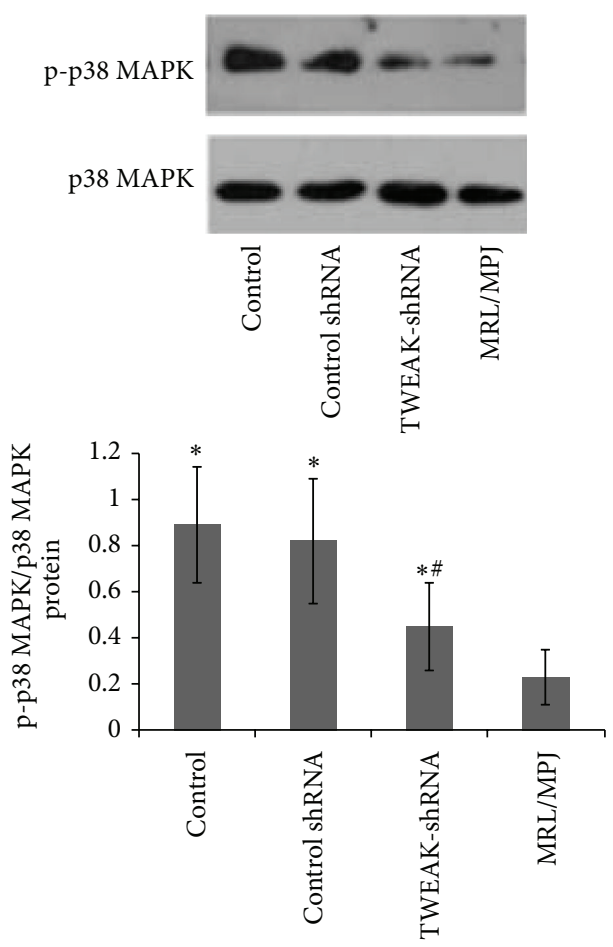

(b)
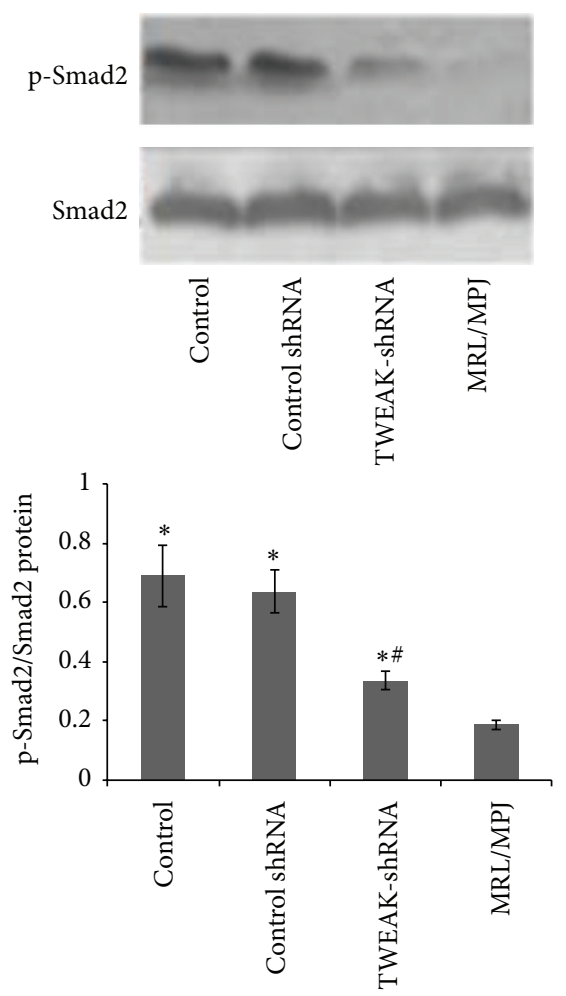

(c)

FIGURE 5: Effects of LV-TWEAK-shRNA treatment on expressions of TGF- $\beta 1$, p-p38 MAPK, and p-Smad2 proteins in kidneys of MRL/lpr mice. Total lysates of kidneys were collected for Western blotting to measure TGF- $\beta 1$ (a), p-p38 MAPK (b), and p-Smad2 (c) protein expressions in MRL/lpr mice. The representative experiments were presented at the top. The ratios of TWEAK/ $\beta$-actin IOD, p-p38 MAPK/p38 MAPK IOD, and p-Smad2/Smad2 IOD were shown at the bottom. Control: MRL/lpr mice treated with PBS; Control shRNA: MRL/lpr mice treated with LV-Control shRNA; TWEAK-shRNA: MRL/lpr mice treated with LV-TWEAK-shRNA; and MRL/MPJ: MRL/MPJ mice as normal control. The error bars represent the standard deviation. ${ }^{*} p<0.01$ versus control MRL/lpr mice and ${ }^{*} p<0.01$ versus MRL/MPJ mice. 


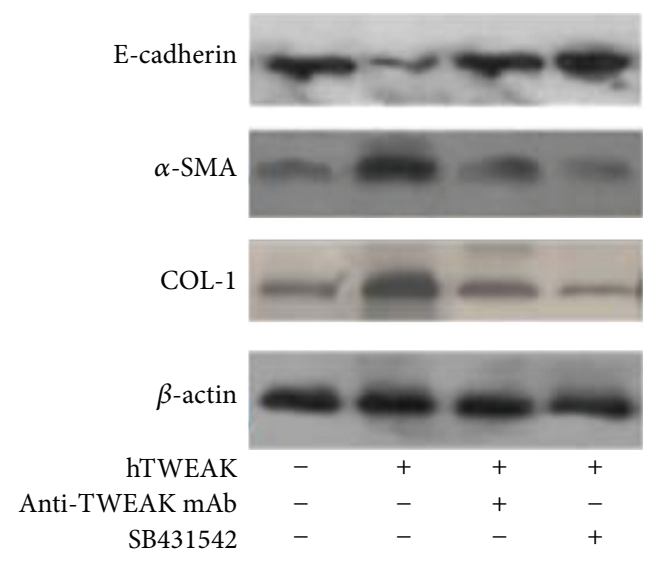

(a)

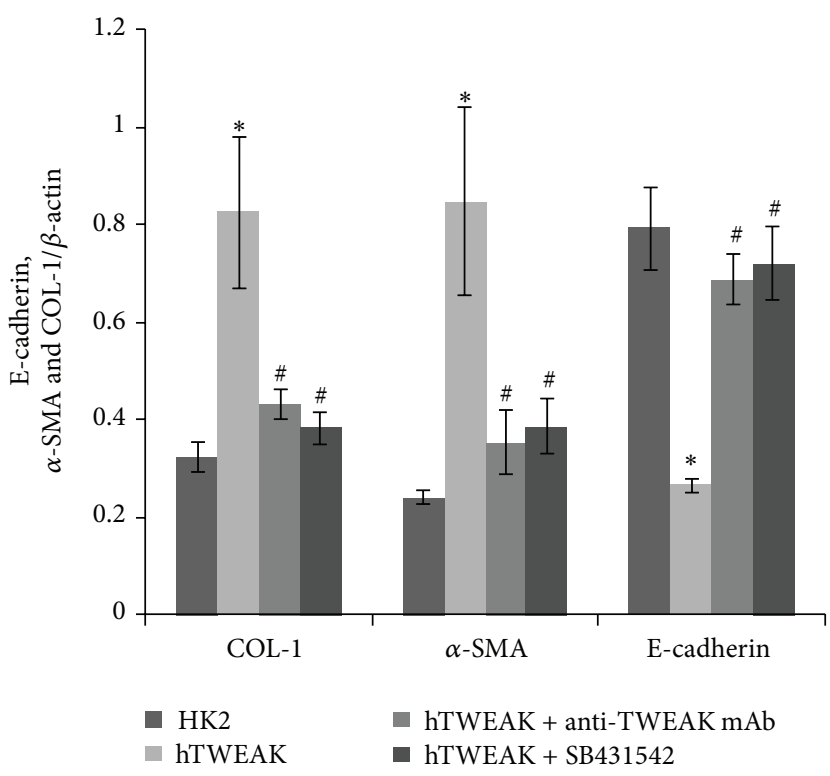

(b)

FIGURE 6: Effects of SB431542 on hTWEAK induced expressions of $\alpha$-SMA, COL-1, and E-cadherin proteins in HK2 cells. Total cell lysates were collected for Western blotting to measure $\alpha$-SMA, COL-1, and E-cadherin protein expressions. The representative experiment was presented in (a). The ratios of $\alpha$-SMA/ $\beta$-actin IOD, COL- $1 / \beta$-actin IOD, and E-cadherin/ $\beta$-actin IOD were shown in (b). HK2: HK2 cells cultured without stimulation; hTWEAK: HK2 cells cultured with hTWEAK; hTWEAK+anti-TWEAK mAb: HK2 cells cocultured with hTWEAK and anti-TWEAK mAb; and hTWEAK+SB431542: HK2 cells cocultured with hTWEAK and SB431542. The error bars represent the standard deviation. Each experiment was repeated at least 3 times. $^{*} p<0.01$ versus HK2 cells cultured without stimulation and ${ }^{\#} p<0.01$ versus HK2 cells cultured with hTWEAK.

expression, accompanied with significantly reductive interstitial and glomerular mesangial matrix deposition, glomerulosclerosis, and interstitial fibrosis in the kidney. Thus we reported that the gene silence of TWEAK alleviated the renal fibrosis of chronic lupus nephritis.

Accumulating evidence suggests that epithelial mesenchymal transition (EMT) is the main process contributing to fibrosis induction [35]. Previous studies have demonstrated that loss of E-cadherin is a critical step in EMT. E-Cadherin is a central component of cell adhesion junctions and is required for epithelial formation and maintaining epithelial homeostasis [31]. The appearance of $\alpha$-SMA in transdifferentiating cells is a hallmark of myofibroblast formation [36]. High renal COL-1 expression is a strong predictor of adverse renal outcome in patients with LN [29]. So we also detected the expressions of $\alpha$-SMA and COL- 1 proteins in the lupus mice. We found that $\alpha$-SMA and COL-1 protein levels increased in kidneys of MRL/lpr mice, and the increase was reversed by LV-TWEAK-shRNA treatment. In in vitro study, we observed higher expression levels of $\alpha$-SMA and COL-1 proteins and lower expression levels of E-cadherin protein in hTWEAK-stimulated HK2 cells compared with control HK2 cells. It has been reported that overproduction and deposition of ECM components, such as COL-1, progressively replace normal parenchyma and disrupt tissue morphology and function [37]. Considered together, these findings demonstrated that TWEAK could accelerate progression of renal fibrosis in lupus mice through EMT. These findings were consistent with reports from other investigators [38-40]; they have reported that female Fn14-knockout MRL-lpr/lpr mice had significantly low levels of proteinuria and ameliorative kidney proliferative changes in lupus nephritis [41]. Recent studies also showed that TWEAK was an inducer of constitutive TGF- $\beta 1, \mathrm{PKG}$, and ERK pathway activation in kidney cells or UUO rats model [10], but the exact mechanism of TWEAK in renal fibrosis in lupus nephritis remains unclear.

As a member of the tumor necrosis factor superfamily ligands, TWEAK has been shown to play an important role in many autoimmune diseases including LN [42]. We found that LV-TWEAK-shRNA treatment downregulated the upregulation of renal TGF- $\beta 1$ protein in MRL/lpr mice; this was also confirmed by in vitro study. hTWEAK stimulation resulted in a significant increased TGF- $\beta 1$ protein expression in HK2 cells, and the upregulation was blocked by coculture with anti-TWEAK antibody. Highly expressed $\alpha$-SMA and COL-1 protein and lowly expressed E-cadherin in the HK2 cells by hTWEAK stimulation were also diminished by antiTWEAK mAb or TGF- $\beta$ receptor 1 inhibitor (SB431542) treatment. As TGF- $\beta 1$ is known to play an important role in renal fibrosis $[43,44]$, we speculated that TWEAK may participate in renal fibrosis in lupus nephritis through TGF$\beta 1$ signaling pathway.

In our previous study, we have found that high expression of TWEAK in PBMCs of lupus nephritis patients could activate the p38 MAPK signaling pathway [45]. In addition to induction of leukocyte recruitment to sites of inflammation [45], p38 MAPK is presumed to be involved in cell proliferation induced by TGF- $\beta[46,47]$ and in TGF- $\beta 1$ induced 


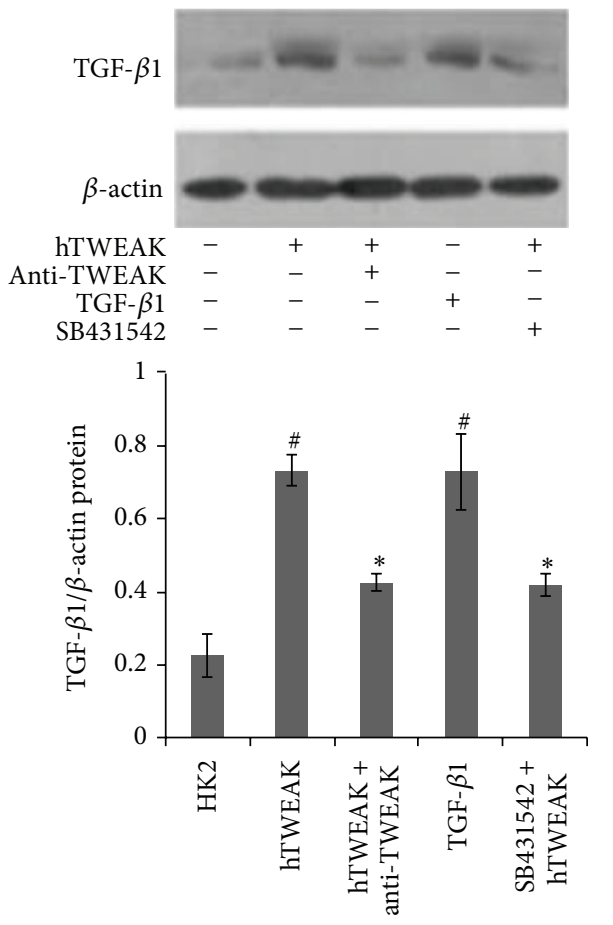

(a)

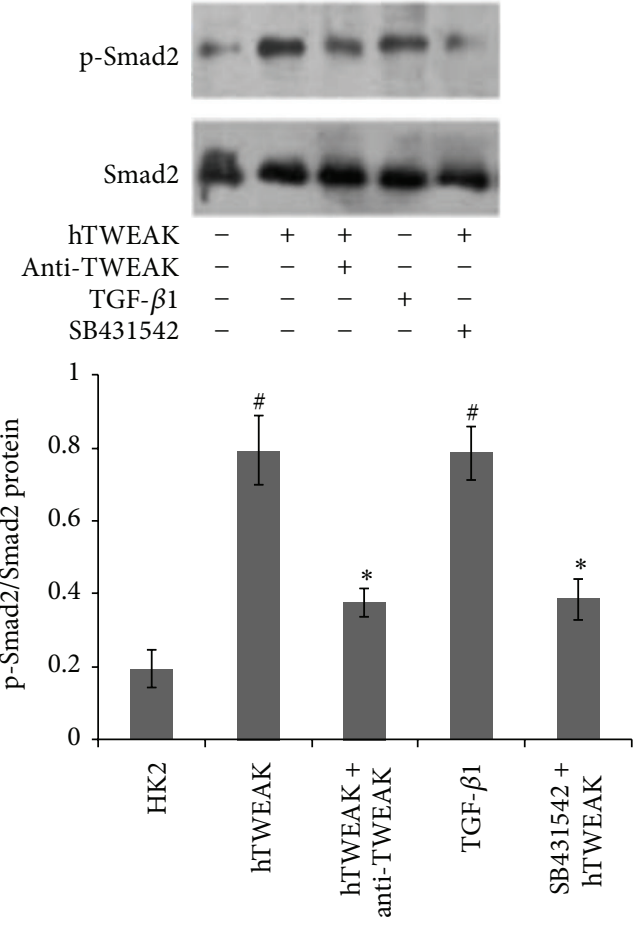

(b)

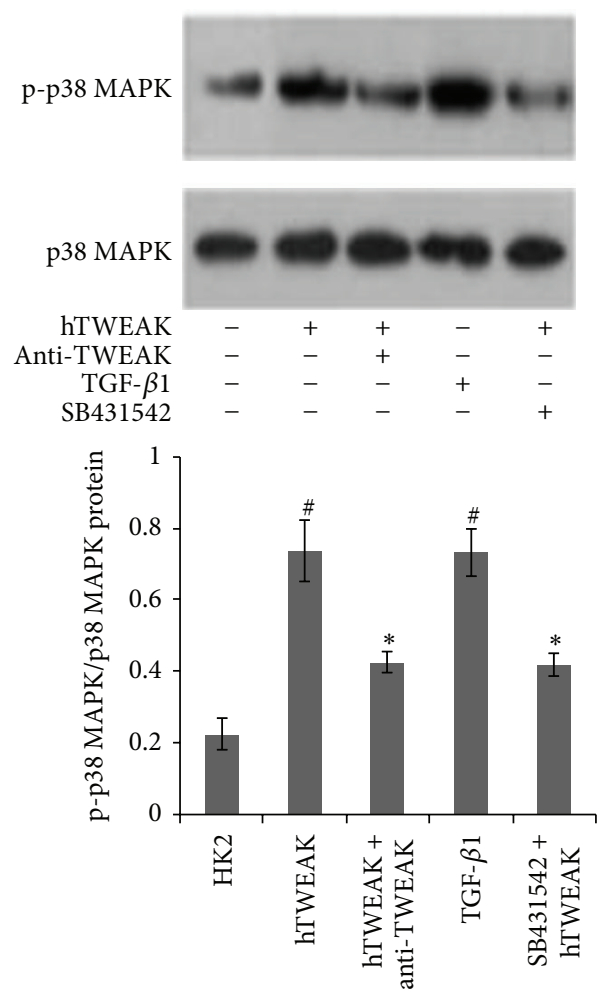

(c)

Figure 7: Effects of SB431542 on hTWEAK induced expressions of TGF- $\beta 1$, p-smad2, and p-p38 MAPK proteins in HK2 cells. Total cell lysates were collected for Western blotting to measure TGF- $\beta 1$ (a), p-Smad2 (b), and p-p38 MAPK (c) protein expressions. The representative experiments were presented at the top. The ratios of TWEAK/ $\beta$-actin IOD, p-p38 MAPK/p38 MAPK IOD, and p-Smad2/Smad2 IOD were shown at the bottom. HK2: HK2 cells cultured without stimulation; hTWEAK: HK2 cells cultured with hTWEAK; hTWEAK+anti-TWEAK: HK2 cells cocultured with hTWEAK and anti-TWEAK mAb; TGF- $\beta 1$ : HK2 cells cultured with TGF- $\beta 1$; and hTWEAK+SB431542: HK2 cells cultured with hTWEAK and SB431542. The error bars represent the standard deviation. Each experiment was repeated at least 3 times. ${ }^{\#} p<0.01$ versus HK2 cells cultured without stimulation and ${ }^{*} p<0.01$ versus HK2 cells cultured with hTWEAK. 
collagen expression [48]. Previous studies have shown that p38 MAPK is one of the important Smad-independent pathways of TGF- $\beta$ stimulation $[49,50]$. Both of the Smad and MAPK pathways have pivotal roles in TGF- $\beta$ signaling regulated renal fibrosis [51]. In these studies, we also observed that the increased protein levels of p-p38 MAPK and pSmad2 in kidneys of MRL/lpr mice were downregulated by LV-TWEAK-shRNA treatment. We further cultured the HK2 cells with TGF- $\beta 1$; the studies revealed significant phosphorylation of p38 MAPK and Smad2 proteins as in lupus mice. hTWEAK treatment had the same effects as TGF- $\beta 1$ on expressions of p-p38 MAPK and p-Smad2 proteins, and TGF$\beta 1$ protein in HK2 cells was also upregulated by hTWEAK stimulation. Moreover, both of TGF- $\beta$ receptor 1 inhibitor SB431542 and anti-TWEAK mAb inhibited phosphorylation of Smad2 and p38 MAPK proteins induced by hTWEAK treatment, and anti-TWEAK mAb also decreased TGF$\beta 1$ protein expression upregulated by hTWEAK treatment. Taken together, we presume that TWEAK mediated positive regulation of TGF- $\beta$ signaling may have a promoting role in kidneys remodeling in lupus nephritis; the phosphorylation of p38 MAPK and Smad2 might be also included in the downstream of TWEAK-TGF- $\beta$ signaling pathway.

In conclusion, TWEAK may contribute to the pathogenesis of kidney remodeling through activating TGF- $\beta$ signaling by inducing the phosphorylation of Smad2 and p38 MAPK proteins in lupus mice. Therefore, blocking TWEAK-TGF- $\beta 1$ signaling pathway may be a promising therapeutic approach in lupus nephritis.

\section{Competing Interests}

There is no conflict of interests among the authors.

\section{Authors' Contributions}

Zhiqin Liu and Leixi Xue equally contributed to the work.

\section{Acknowledgments}

This work was partly supported by the Suzhou Science and Technology Development Project (Grant no. SYS201231) and the National Natural Science Foundation of China (Grant no. 81200507).

\section{References}

[1] A. T. Borchers, C. A. Aoki, S. M. Naguwa, C. L. Keen, Y. Shoenfeld, and M. E. Gershwin, "Neuropsychiatric features of systemic lupus erythematosus," Autoimmunity Reviews, vol. 4, no. 6, pp. 329-344, 2005.

[2] P. Boor, T. Ostendorf, and J. Floege, "Renal fibrosis: novel insights into mechanisms and therapeutic targets," Nature Reviews Nephrology, vol. 6, no. 11, pp. 643-656, 2010.

[3] Y. Xia, S. R. Campbell, A. Broder et al., "Inhibition of the TWEAK/Fn14 pathway attenuates renal disease in nephrotoxic serum nephritis," Clinical Immunology, vol. 145, no. 2, pp. 108121, 2012.
[4] S. R. Wiley and J. A. Winkles, "TWEAK, a member of the TNF superfamily, is a multifunctional cytokine that binds the TweakR/Fn14 receptor," Cytokine and Growth Factor Reviews, vol. 14, no. 3-4, pp. 241-249, 2003.

[5] S. R. Wiley, L. Cassiano, T. Lofton et al., "A novel TNF receptor family member binds TWEAK and is implicated in angiogenesis," Immunity, vol. 15, no. 5, pp. 837-846, 2001.

[6] A. B. Sanz, M. D. Sanchez-Niño, and A. Ortiz, "TWEAK, a multifunctional cytokine in kidney injury," Kidney International, vol. 80, no. 7, pp. 708-718, 2011.

[7] A. B. Sanz, M. D. Sanchez-Niño, C. Martín-Cleary, A. Ortiz, and A. M. Ramos, "Progress in the development of animal models of acute kidney injury and its impact on drug discovery," Expert Opinion on Drug Discovery, vol. 8, no. 7, pp. 879-895, 2013.

[8] Y. Itoigawa, N. Harada, S. Harada et al., "TWEAK enhances TGF- $\beta$-induced epithelial-mesenchymal transition in human bronchial epithelial cells," Respiratory Research, vol. 16, article 48, 2015.

[9] T. Dohi and L. C. Burkly, "The TWEAK/Fn14 pathway as an aggravating and perpetuating factor in inflammatory diseases; focus on inflammatory bowel diseases," Journal of Leukocyte Biology, vol. 92, no. 2, pp. 265-279, 2012.

[10] A. C. Ucero, A. Benito-Martin, I. Fuentes-Calvo et al., "TNFrelated weak inducer of apoptosis (TWEAK) promotes kidney fibrosis and Ras-dependent proliferation of cultured renal fibroblast," Biochimica et Biophysica Acta-Molecular Basis of Disease, vol. 1832, no. 10, pp. 1744-1755, 2013.

[11] Z. Zhao, L. C. Burkly, S. Campbell et al., "TWEAK/Fn14 interactions are instrumental in the pathogenesis of nephritis in the chronic graft-versus-host model of systemic lupus erythematosus," The Journal of Immunology, vol. 179, no. 11, pp. 79497958, 2007.

[12] N. Schwartz, L. Su, L. C. Burkly et al., "Urinary TWEAK and the activity of lupus nephritis," Journal of Autoimmunity, vol. 27, no. 4, pp. 242-250, 2006.

[13] N. Schwartz, T. Rubinstein, L. C. Burkly et al., "Urinary TWEAK as a biomarker of lupus nephritis: a multicenter cohort study," Arthritis Research and Therapy, vol. 11, no. 5, article R143, 2009.

[14] T. Dohi, A. Borodovsky, P. Wu et al., "TWEAK/Fn14 pathway: a nonredundant role in intestinal damage in mice through a TWEAK/intestinal epithelial cell axis," Gastroenterology, vol. 136, no. 3, pp. 912-923, 2009.

[15] H. Li, A. Mittal, P. K. Paul et al., “Tumor necrosis factor-related weak inducer of apoptosis augments matrix metalloproteinase 9 (MMP-9) production in skeletal muscle through the activation of nuclear factor- $\kappa \mathrm{B}$-inducing kinase and p38 mitogenactivated protein kinase: a potential role MMP-9 in myopathy," Journal of Biological Chemistry, vol. 284, no. 7, pp. 4439-4450, 2009.

[16] L. C. Burkly, J. S. Michaelson, K. Hahm, A. Jakubowski, and T. S. Zheng, "TWEAKing tissue remodeling by a multifunctional cytokine: role of TWEAK/Fn14 pathway in health and disease," Cytokine, vol. 40, no. 1, pp. 1-16, 2007.

[17] K. Hotta, M. Sho, I. Yamato et al., "Direct targeting of fibroblast growth factor-inducible 14 protein protects against renal ischemia reperfusion injury," Kidney International, vol. 79, no. 2, pp. 179-188, 2011.

[18] J. Wen, Y. Xia, A. Stock et al., "Neuropsychiatric disease in murine lupus is dependent on the TWEAK/Fn14 pathway," Journal of Autoimmunity, vol. 43, no. 1, pp. 44-54, 2013.

[19] J. Wen, J. Doerner, K. Weidenheim et al., "TNF-like weak inducer of apoptosis promotes blood brain barrier disruption 
and increases neuronal cell death in MRL/lpr mice," Journal of Autoimmunity, vol. 60, no. 1, pp. 40-50, 2015.

[20] A. M. Doerner and B. L. Zuraw, "TGF- $\beta 1$ induced epithelial to mesenchymal transition (EMT) in human bronchial epithelial cells is enhanced by IL-1 $\beta$ but not abrogated by corticosteroids," Respiratory Research, vol. 10, no. 1, article 100, 2009.

[21] Y. Hosokawa, I. Hosokawa, K. Ozaki, H. Nakae, and T. Matsuo, "Proinflammatory effects of tumour necrosis factor-like weak inducer of apoptosis (TWEAK) on human gingival fibroblasts," Clinical and Experimental Immunology, vol. 146, no. 3, pp. 540549, 2006.

[22] N. Ebihara, M. Nakayama, T. Tokura, M. Iwatsu, H. Ushio, and A. Murakami, "Proinflammatory effect of TWEAK/Fn14 interaction in human retinal pigment epithelial cells," Current Eye Research, vol. 34, no. 10, pp. 836-844, 2009.

[23] A. Moustakas, S. Souchelnytskyi, and C.-H. Heldin, "Smad regulation in TGF- $\beta$ signal transduction," Journal of Cell Science, vol. 114, no. 24, pp. 4359-4369, 2001.

[24] R. Kalluri and E. G. Neilson, "Epithelial-mesenchymal transition and its implications for fibrosis," Journal of Clinical Investigation, vol. 112, no. 12, pp. 1776-1784, 2003.

[25] C. Stambe, R. C. Atkins, G. H. Tesch, T. Masaki, G. F. Schreiner, and D. J. Nikolic-Paterson, "The role of p38 $\alpha$ mitogen-activated protein kinase activation in renal fibrosis," Journal of the American Society of Nephrology, vol. 15, no. 2, pp. 370-379, 2004.

[26] Y. Liu, "Renal fibrosis: new insights into the pathogenesis and therapeutics," Kidney International, vol. 69, no. 2, pp. 213-217, 2006.

[27] Z.-C. Liu, Q.-L. Zhou, Z.-Q. Liu, X.-Z. Li, X.-X. Zuo, and R. Tang, "Tumor necrosis factor-like weak inducer of apoptosis (TWEAK) mediates p38 mitogen-activated protein kinase activation and signal transduction in peripheral blood mononuclear cells from patients with lupus nephritis," Inflammation, vol. 35, no. 3, pp. 935-943, 2012.

[28] D. L. Feldman, T. C. Mogelesky, M. Chou, and A. Y. Jeng, "Enhanced expression of renal endothelin-converting enzyme1 and endothelin-A-receptor mRNA in rats with interstitial fibrosis following ureter ligation," Journal of Cardiovascular Pharmacology, vol. 36, no. 5, pp. S255-S259, 2000.

[29] C. Tachaudomdach, S. Kantachuvesiri, S. Wongpraphairot et al., "High collagen I gene expression as an independent predictor of adverse renal outcomes in lupus nephritis patients with preserved renal function," Archives of Pathology and Laboratory Medicine, vol. 139, no. 3, pp. 378-387, 2015.

[30] M. Zeisberg, F. Strutz, and G. A. Müller, "Role of fibroblast activation in inducing interstitial fibrosis," Journal of Nephrology, vol. 13, no. 3, pp. S111-S120, 2000.

[31] T. Lecuit and A. S. Yap, "E-cadherin junctions as active mechanical integrators in tissue dynamics," Nature Cell Biology, vol. 17, no. 5, pp. 533-539, 2015.

[32] L. Gu, A. Weinreb, X.-P. Wang et al., "Genetic determinants of autoimmune disease and coronary vasculitis in the MRLlpr/lpr mouse model of systemic lupus erythematosus," Journal of Immunology, vol. 161, no. 12, pp. 6999-7006, 1998.

[33] M.-L. Santiago-Raber, C. Laporte, L. Reininger, and S. Izui, "Genetic basis of murine lupus," Autoimmunity Reviews, vol. 3, no. 1, pp. 33-39, 2004.

[34] S. Desplat-Jégo, R. Creidy, S. Varriale et al., "Anti-TWEAK monoclonal antibodies reduce immune cell infiltration in the central nervous system and severity of experimental autoimmune encephalomyelitis," Clinical Immunology, vol. 117, no. 1, pp. 15-23, 2005.
[35] N.-H. Myong, "Loss of E-cadherin and acquisition of vimentin in epithelial-mesenchymal transition are noble indicators of uterine cervix cancer progression," Korean Journal of Pathology, vol. 46, no. 4, pp. 341-348, 2012.

[36] L. Ding, Z. Zhang, D. Shang et al., " $\alpha$-Smooth muscle actin-positive myofibroblasts, in association with epithelialmesenchymal transition and lymphogenesis, is a critical prognostic parameter in patients with oral tongue squamous cell carcinoma," Journal of Oral Pathology and Medicine, vol. 43, no. 5, pp. 335-343, 2014.

[37] T. A. Wynn, "Cellular and molecular mechanisms of fibrosis," The Journal of Pathology, vol. 214, no. 2, pp. 199-210, 2008.

[38] T. Dohi, A. Borodovsky, P. Wu et al., “TWEAK/Fn14 pathway: a nonredundant role in intestinal damage in mice through a TWEAK/intestinal epithelial cell axis," Gastroenterology, vol. 136, no. 3, pp. 912-923.e8, 2009.

[39] H. Li, A. Mittal, P. K. Paul et al., "Tumor necrosis factorrelated weak inducer of apoptosis augments matrix metalloproteinase 9 (MMP-9) production in skeletal muscle through the activation of nuclear factor- $\kappa \mathrm{B}$-inducing kinase and p38 mitogen-activated protein kinase: a potential role of MMP-9 in myopathy," The Journal of Biological Chemistry, vol. 284, no. 7, pp. 4439-4450, 2009.

[40] B. Muñoz-Garca, J. Madrigal-Matute, J. A. Moreno et al., "TWEAK-Fn14 interaction enhances plasminogen activator inhibitor 1 and tissue factor expression in atherosclerotic plaques and in cultured vascular smooth muscle cells," Cardiovascular Research, vol. 89, no. 1, pp. 225-233, 2011.

[41] Y. Xia, L. C. Herlitz, S. Gindea et al., "Deficiency of fibroblast growth factor-inducible 14 (Fn14) preserves the filtration barrier and ameliorates lupus nephritis," Journal of the American Society of Nephrology, vol. 26, no. 5, pp. 1053-1070, 2015.

[42] J. A. Winkles, "The TWEAK-Fn14 cytokine-receptor axis: discovery, biology and therapeutic targeting," Nature Reviews Drug Discovery, vol. 7, no. 5, pp. 411-425, 2008.

[43] Z. Kassiri, G. Y. Oudit, V. Kandalam et al., "Loss of TIMP3 enhances interstitial nephritis and fibrosis," Journal of the American Society of Nephrology, vol. 20, no. 6, pp. 1223-1235, 2009.

[44] M. Giannandrea and W. C. Parks, "Diverse functions of matrix metalloproteinases during fibrosis," DMM Disease Models and Mechanisms, vol. 7, no. 2, pp. 193-203, 2014.

[45] D. C. Underwood, R. R. Osborn, S. Bochnowicz et al., "SB 239063, a p38 MAPK inhibitor, reduces neutrophilia, inflammatory cytokines, MMP-9, and fibrosis in lung," American Journal of Physiology - Lung Cellular and Molecular Physiology, vol. 279, no. 5, pp. L895-L902, 2000.

[46] Y. Fu, L. M. O'Connor, T. G. Shepherd, and M. W. Nachtigal, “The p38 MAPK inhibitor, PD169316, inhibits transforming growth factor $\beta$-induced Smad signaling in human ovarian cancer cells," Biochemical and Biophysical Research Communications, vol. 310, no. 2, pp. 391-397, 2003.

[47] M. Takekawa, K. Tatebayashi, F. Itoh, M. Adachi, K. Imai, and H. Saito, "Smad-dependent GADD $45 \beta$ expression mediates delayed activation of p38 MAP kinase by TGF- $\beta$," The EMBO Journal, vol. 21, no. 23, pp. 6473-6482, 2002.

[48] B. Y. Chin, A. Mohsenin, S. X. Li, A. M. K. Choi, and M. E. Choi, "Stimulation of pro- $\alpha 1$ (I) collagen by TGF- $\beta 1$ in mesangial cells: role of the p38 MAPK pathway," American Journal of Physiology: Renal Physiology, vol. 280, no. 3, pp. F495-F504, 2001.

[49] Y. Sano, J. Harada, S. Tashiro, R. Gotoh-Mandeville, T. Maekawa, and S. Ishii, "ATF-2 is a common nuclear target 
of smad and TAK1 pathways in transforming growth factor- $\beta$ signaling," Journal of Biological Chemistry, vol. 274, no. 13, pp. 8949-8957, 1999.

[50] H. Hanafusa, J. Ninomiya-Tsuji, N. Masuyama et al., "Involvement of the p38 mitogen-activated protein kinase pathway in transforming growth factor- $\beta$-induced gene expression," The Journal of Biological Chemistry, vol. 274, no. 38, pp. 27161-27167, 1999.

[51] K. Miyazono, P. Ten Dijke, and C.-H. Heldin, "TGF- $\beta$ signaling by Smad proteins," Advances in Immunology, vol. 75, no. 1, pp. 115-157, 2000. 


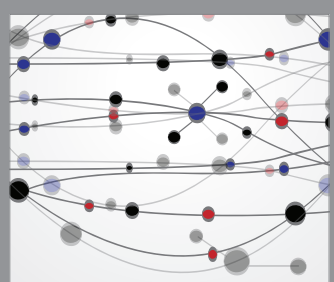

The Scientific World Journal
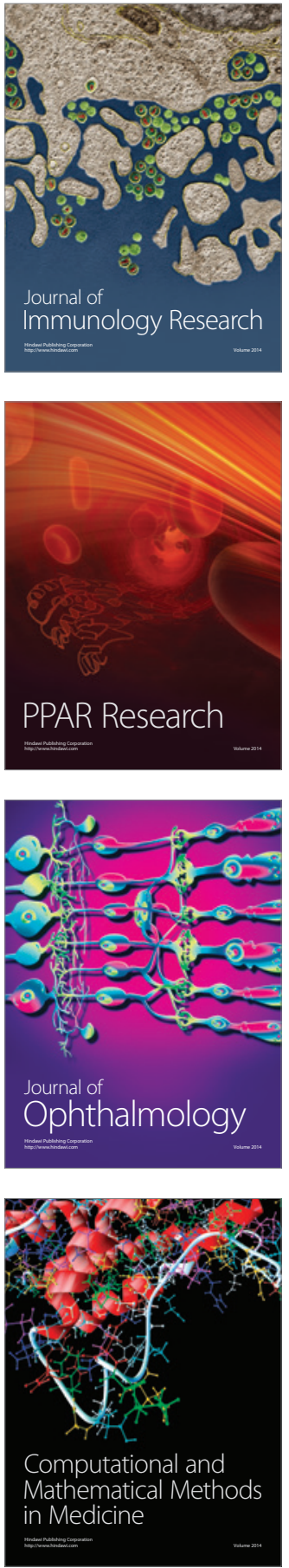

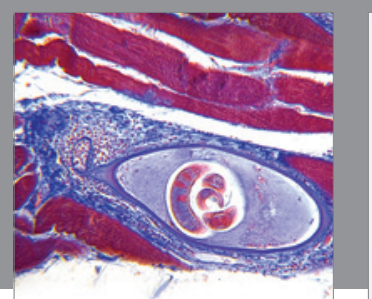

Gastroenterology Research and Practice

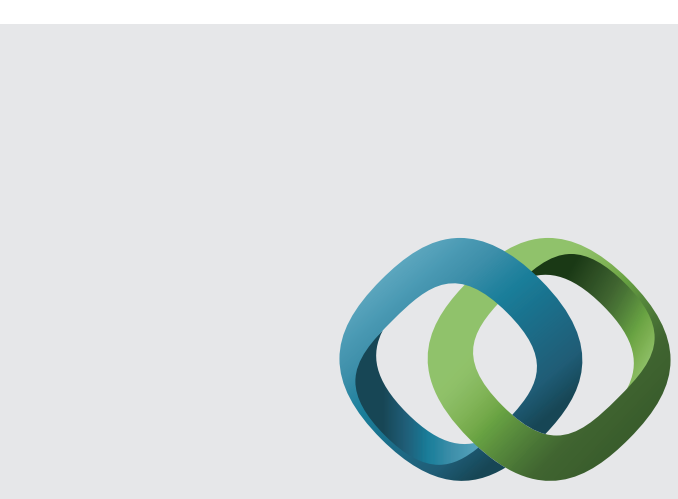

\section{Hindawi}

Submit your manuscripts at

http://www.hindawi.com
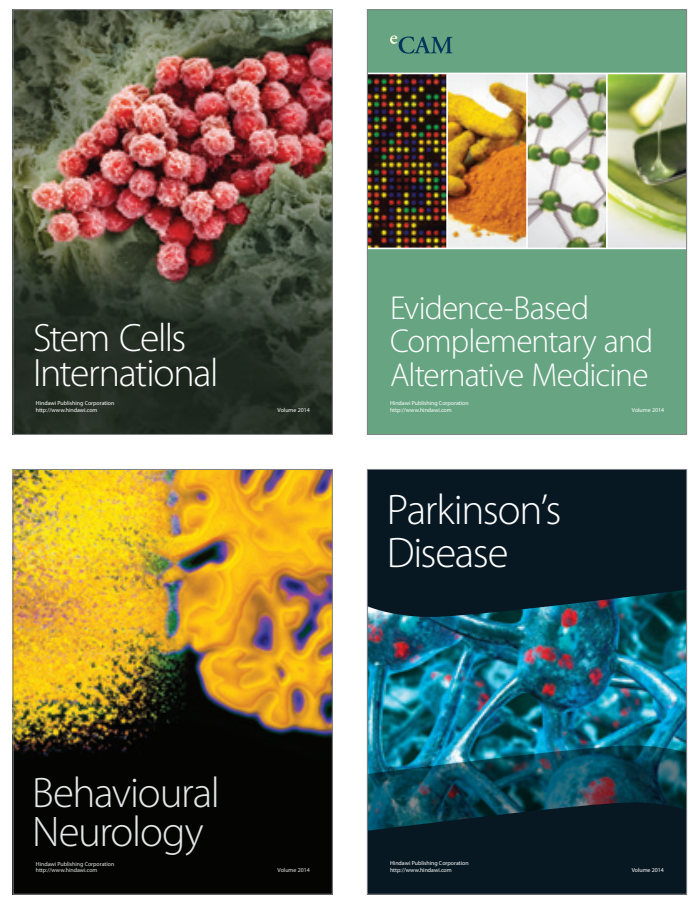
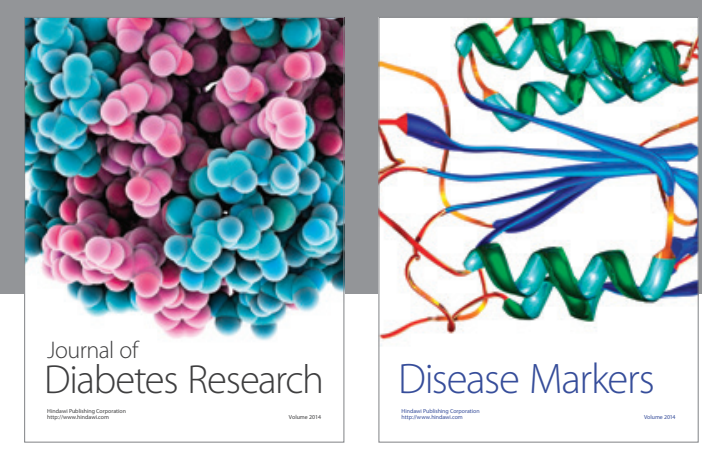

Disease Markers
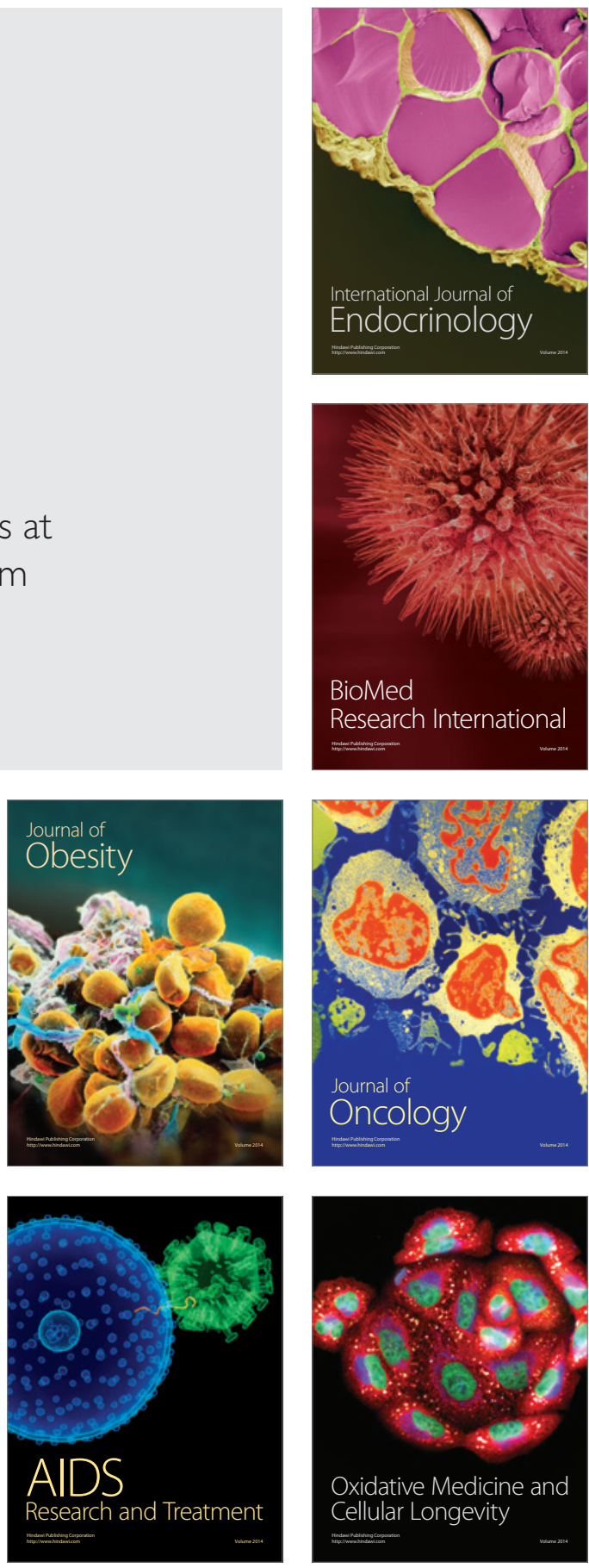